\title{
Adherence to healthy lifestyle and risk of gestational diabetes mellitus: prospective cohort study
}

\author{
(c) (1) (8) OPEN ACCESS
}

\author{
Cuilin Zhang investigator ${ }^{1}$, Deirdre K Tobias research fellow ${ }^{2}$, Jorge E Chavarro assistant professor ${ }^{23}$, \\ Wei Bao postdoctoral fellow ${ }^{1}$, Dong Wang research fellow ${ }^{2}$, Sylvia H Ley research fellow ${ }^{2}$, Frank \\ B Hu professor ${ }^{23}$
}

'Division of Intramural Population Health Research, Eunice Kennedy Shriver National Institute of Child Health and Human Development, National Institutes of Health, Bethesda, MD 20852, USA; ${ }^{2}$ Departments of Nutrition and Epidemiology, Harvard School of Public Health, Boston, MA 02115, USA ${ }^{3}$ Channing Division of Network Medicine, Department of Medicine, Brigham and Women's Hospital and Harvard Medical School, Boston, MA 02115, USA

\begin{abstract}
Objective To quantify the association between a combination of healthy lifestyle factors before pregnancy (healthy body weight, healthy diet, regular exercise, and not smoking) and the risk of gestational diabetes.

Design Prospective cohort study.

Setting Nurses' Health Study II, United States.

Participants 20136 singleton live births in 14437 women without chronic disease.

Main outcome measure Self reported incident gestational diabetes diagnosed by a physician, validated by medical records in a previous study.

Results Incident first time gestational diabetes was reported in 823 pregnancies. Each lifestyle factor measured was independently and significantly associated with risk of gestational diabetes. The combination of three low risk factors (non-smoker, $\geq 150$ minutes a week of moderate to vigorous physical activity, and healthy eating (top two fifths of Alternate Healthy Eating Index-2010 adherence score)) was associated with a $41 \%$ lower risk of gestational diabetes compared with all other pregnancies (relative risk $0.59,95 \%$ confidence interval 0.48 to 0.71 ). Addition of body mass index (BMI) $<25$ before pregnancy (giving a combination of four low risk factors) was associated with a $52 \%$ lower risk of gestational diabetes compared with all other pregnancies (relative risk $0.48,0.38$ to 0.61 ). Compared with pregnancies in women who did not meet any of the low risk lifestyle factors, those meeting all four criteria had an $83 \%$ lower risk of gestational diabetes (relative risk $0.17,0.12$ to 0.25 ). The population attributable risk percentage of the four risk factors in combination (smoking, inactivity, overweight, and poor diet) was $47.5 \%$ (95\% confidence interval $35.6 \%$ to $56.6 \%$ ). A similar population attributable risk percentage $(49.2 \%)$ was observed when the
\end{abstract}

distributions of the four low risk factors from the US National Health and Nutrition Examination Survey (2007-10) data were applied to the calculation.

Conclusions Adherence to a low risk lifestyle before pregnancy is associated with a low risk of gestational diabetes and could be an effective strategy for the prevention of gestational diabetes.

\section{Introduction}

Gestational diabetes, defined as glucose intolerance with onset or first recognition in pregnancy, is one of the most common complications in pregnancy. ${ }^{1}$ In the United States, gestational diabetes affects $1.1-25.5 \%$ of pregnancies depending on the criteria used. ${ }^{2}$ It represents a growing health concern; multiple population based studies have shown increases in the occurrence of gestational diabetes in the past decade..$^{3-5}$ It is linked to short term and long term adverse health outcomes in women and their offspring. ${ }^{16}$ For instance, women with gestational diabetes have an increased risk for gestational hypertension and pre-eclampsia during pregnancy ${ }^{7}$ and an exceptionally high risk for type 2 diabetes after pregnancy. ${ }^{8}$ High glucose concentrations in pregnancy have long been regarded as a teratogen, affecting fetal structure, function, and development, and as having long term adverse impacts on the offspring. Children born after a pregnancy affected by gestational diabetes are more likely to be born macrosomic and to have birth defects. ${ }^{9}$ Moreover, there is evidence to suggest that they are also at higher risk of developing childhood obesity ${ }^{10}$ and glucose intolerance in early adulthood, ${ }^{11}$ although findings have been inconsistent, and females are more likely to develop gestational diabetes themselves, ${ }^{12}$ leading to a trans-generational cycle of 
diabetes-begets-diabetes. Prevention of gestational diabetes could be an important strategy in curbing the obesity and diabetes epidemic in this and future generations. Therefore, it is pivotal to identify potentially modifiable risk factors and to quantify their potential impact on this common condition.

Several potentially modifiable factors before pregnancy have been related to a lower risk of gestational diabetes. ${ }^{13}$ These include maintaining a healthy body weight, adapting a healthy diet, regular physical activity, and abstinence from cigarette smoking. ${ }^{14-16}$ We examined the joint effect of lifestyle characteristics on risk of gestational diabetes. We hypothesized that these factors are jointly associated with a substantially lower risk of gestational diabetes independent of other major risk factors such as advanced maternal age, greater parity, and family history of diabetes.

\section{Methods}

\section{Source population}

The study was based on the Nurses' Health Study II, an ongoing prospective cohort that was established in 1989 by enrolling 116671 female nurses aged 24-44 at baseline..$^{14}$ Questionnaires are administered biennially to update lifestyle characteristics and health related outcomes. The 1989 baseline questionnaire collected information on medical, diagnostic, and prescription history, date of birth, occupational status, family history of diabetes, gravidity, height and weight, and a range of other characteristics. Time varying characteristics are updated every two years.

After 2001, most participants had passed the usual reproductive age (all were aged $\geq 35,70 \%$ were aged $\geq 40$, and $20 \%$ aged $\geq 45$ ) and information on the diagnosis of gestational diabetes was not collected in the questionnaire. We included in the analysis individual singleton live births up to the end of 2001 if the participant did not report gestational diabetes in a previous pregnancy, a diagnosis of type 2 diabetes or cancer, or a cardiovascular disease event (myocardial infarction, stroke, coronary artery bypass graft procedure, or angina) before an otherwise eligible pregnancy (see appendix 1). Pregnancies after gestational diabetes were not included because women with gestational diabetes in a previous pregnancy were likely to change their diet and lifestyle during the next pregnancy to prevent a recurrence. ${ }^{17} \mathrm{We}$ conducted sensitivity analyses with all pregnancies regardless of previous gestational diabetes status. Follow-up for each questionnaire cycle is $>90 \%$ through 2001 .

\section{Ascertainment of lifestyle factors}

Twice a year we obtained information on participants' smoking status and weight. Self reported weight was highly correlated with weight measured by a technician among a random subset of participants in the Boston area cohort $(\mathrm{r}=0.97){ }^{18}$

Beginning in 1991 and every four years thereafter, participants were asked to complete a semiquantitative food frequency questionnaire in addition to the main questionnaire. The food frequency questionnaire is designed to measure average dietary intakes over the past year and has been extensively validated. ${ }^{19} 20$ To characterize overall dietary quality, we created a summary diet score based on the Alternate Healthy Eating Index-2010, ${ }^{21}$ which is based on the US Department of Agriculture Healthy Eating Index, designed to measure adherence to US dietary guidelines. ${ }^{22} \mathrm{We}$ computed scores for adherence to the index before pregnancy from each food frequency questionnaire cycle occurring before a given pregnancy. We included 10 of 11 the components of the index in our diet score: higher intakes of vegetables, fruit, nuts, whole grains, polyunsaturated fatty acids, and long chain omega 3 fatty acids and lower intakes of red and processed meats, sugar sweetened beverages, trans fats, and sodium. Alcohol, a component of the Alternate Healthy Eating Index-2010, was not included in this analysis because of the concern of its potential adverse impact on fetal outcomes. Participants' scores for each of the individual components ranged from 0 to 10 based on level of intake, with 10 representing optimal dietary behavior. The overall diet score ranged from 2.5 (worst) to 77.5 (best). Pregnancies were excluded if the participant did not return a prepregnancy food frequency questionnaire, left more than 70 food frequency questionnaire items blank, or reported unrealistic total daily energy intakes (<2090 kJ (<500 kcal) or $14630 \mathrm{~kJ}$ (>3500 kcal)).

Physical activity was assessed on the main questionnaire in 1989, 1991, and 1997. Participants were asked to report the average amount of time they spent on each of the following moderate to vigorous recreational activities each week: bicycling, calisthenics or use of a rowing machine, lap swimming, squash or racquetball, jogging, running, tennis, or other aerobic exercise. Women were also asked to report the amount of time they spent each week walking (that is, walking or hiking outdoors, including walking to work) and to categorize their usual walking pace outdoors as easy or casual (slower than $2.0 \mathrm{mph}$ ), normal (2.0-2.9 mph), brisk (3.0-3.9 mph), or very brisk or striding (faster than $4.0 \mathrm{mph}$ ). Usual total physical activity before pregnancy was calculated as the sum of the minutes spent each week in each activity, including brisk or very brisk walking. Validity and reproducibility of the questionnaire were evaluated and reported previously. ${ }^{23}$ Briefly, the correlation between physical activity as reported in one week recalls and that reported on the questionnaires was $r=0.79$. The correlation between moderate to vigorous activity reported in diaries and that reported on the questionnaires was $r=0.62$.

\section{Outcome assessment}

The outcome of interest was incident gestational diabetes. Gestational diabetes diagnosed by a physician was ascertained by self report on each biennial questionnaire through 2001 . Investigators ceased the update of occurrence of gestational diabetes in 2001 as most women had passed reproductive age. In a validation study among a subgroup of the Nurses' Health Study II cohort, $94 \%$ of self reported gestational diabetes events were confirmed by medical records. ${ }^{14}{ }^{24}$ Among the confirmed diagnoses of gestational diabetes, physicians were most likely to use the National Diabetes Data Group criteria. From a supplemental questionnaire sent to a random sample of parous women who did not report gestational diabetes $(n=114), 83 \%$ reported undergoing a $50 \mathrm{~g}$ glucose screening test during pregnancy and $100 \%$ reported frequent prenatal urine glucose screening, suggesting a high level of gestational diabetes surveillance among both cases and non-cases in this cohort.

\section{Definition of the low risk group}

We considered four low risk lifestyle factors before pregnancy based on the strength of the evidence supporting their role in the development of gestational diabetes: a healthy body weight, adherence to a healthy dietary pattern, regular exercise, and abstinence from cigarette smoking. ${ }^{13}{ }^{14}$ Not being overweight or obese was classified as a BMI $<25 .{ }^{25}$ Participants in the upper two fifths for their scores before pregnancy on the Alternate Healthy Eating Index-2010 were considered to be in the lower risk category for adherence to a healthy diet. Low risk usual exercise was defined as an average of at least one half hour a day of vigorous or moderate physical activity for five days a 
week (150 minutes/week), including brisk walking, in keeping with published US guidelines. ${ }^{26}$ Women who reported being current non-smokers in the questionnaire preceding the pregnancy were counted as low risk for smoking status. For each of the four factors, we created a binary variable, with participants receiving 1 if they met the criteria for low risk and 0 otherwise.

\section{Statistical analysis}

Cumulative average amounts of dietary food and levels physical activity before pregnancy were calculated at each time period to reduce variation within participants and reflect long term habitual levels. If a woman was pregnant while she was filling out a given questionnaire, data from the previous questionnaire were carried forward for exposure variables to better represent usual lifestyle before pregnancy, when available. We used multivariable log binomial models (that is, binomial regression models with a log link function) with generalized estimating equations, specifying an exchangeable correlation structure, to estimate the relative risks and $95 \%$ confidence intervals for associations between the four major potentially modifiable factors (smoking status, score on Alternate Healthy Eating Index-2010 (fifths), physical activity (fifths), and BMI) and risk of gestational diabetes. We used generalized estimating equations to account for potential correlations between repeated observations contributed by a single participant (that is, more than one pregnancy). ${ }^{27}$ Covariates in the multivariable models included the above variables, age (continuous), parity (nulliparity or 1, 2, 3, or $\geq 4$ previous pregnancies), race/ethnicity (white, African-American, Hispanic, Asian, other, missing), family history of diabetes (yes, no), history of infertility (yes, no), year of pregnancy, total energy intake (fifths) before pregnancy, and alcohol intake before pregnancy (none, 1-5, $6-15, \geq 16$ g/day).

We calculated the population attributable risk percentages and 95\% confidence intervals to estimate the proportion of gestational diabetes in this cohort that hypothetically would not have occurred if all pregnancies were in the low risk group ${ }^{28}$ by comparing pregnancies in the low risk category (for each factor individually or in combination) with all the others. ${ }^{29}$ To allow valid calculation of the population attributable risk percentage, we estimated the relative risks with age explicitly in the multivariate model based on log binomial generalized estimating equation models, as mentioned above. For simplicity and increased statistical efficiency, we used a single binary categorical variable to calculate the population attributable risk percentage pertaining to the impact of more than one low risk factor. In addition, we derived similar percentages and 95\% confidence intervals based on the distributions of the four low risk factors according to the recent prevalence among women of reproductive ages (ages 18-45) in the nationally representative US National Health and Nutrition Examination Survey (2007-10), which is a program of studies designed to assess the health and nutritional status of adults and children in the US.

We also carried out a sensitivity analysis to assess the robustness of our findings, restricting our analysis to first births from nulliparous women to reduce possible confounding by experiences in previous pregnancies. We evaluated effect modification by established risk factors for gestational diabetes including age $(<35 v \geq 35)$, BMI before pregnancy $(<25 v \geq 25)$, family history of diabetes (yes, no), and race (white $v$ non-white) by conducting stratified analyses. P values for heterogeneity were derived from the cross product interaction term coefficient (exposure variable $\times$ binary effect modifier variable) added to the main effects multivariable model. All statistical analyses were performed with SAS software (version 9.1; SAS Institute). $\mathrm{P}<0.05$ was considered significant.

\section{Results}

During the 10 years of follow-up, we identified 823 pregnancies affected by incident gestational diabetes among the 20136 eligible singleton pregnancies in 14437 women (93\% white). The strongest individual risk factor for gestational diabetes was a higher BMI before pregnancy (table $1 \Downarrow$ ). Even a BMI at the high end of the normal range (23.0-24.9) was associated with a significantly increased risk of gestational diabetes. Each of the other lifestyle factors before pregnancy (total moderate/vigorous physical activity, smoking status, adherence to Alternate Healthy Eating Index-2010 diet) was also significantly associated with risk of gestational diabetes. The associations remained significant after adjustment for other risk factors of gestational diabetes such as maternal age, parity, and family history of diabetes.

When we categorized exposures into "low risk" lifestyle factors, normal weight before pregnancy, regular exercise, healthy diet, and not smoking were all significantly and independently associated with a lower risk of gestational diabetes (table $2 \Downarrow$ ). The population attributable risk percentage $(95 \%$ confidence interval) for overweight/obesity before pregnancy was $28 \%$ ( $25 \%$ to $31 \%$ ) (table 2 ). Independent of BMI, the population attributable risk percentage for not meeting the US physical activity guidelines of 150 minutes of moderate to vigorous physical activity a week and lack of adherence to a healthful dietary pattern was $10 \%$ and $12 \%$, respectively. The corresponding population attributable risk percentage for current smoking was only $3 \%$ as the prevalence of smoking before pregnancy in our population was low (8\%). The population attributable risk percentage (95\% confidence interval) derived based on the distributions of the four risk factors according to the recent prevalence among women of reproductive ages (ages 18-45) in the National Health and Nutrition Examination Survey 2007-10 (see appendix 2) was 6\% (2\% to $10 \%$ ) for current smoking, $33 \%(28,39)$ for being overweight or obese, $11 \%$ ( $3 \%$ to $19 \%$ ) for lack of adherence to a healthy diet, and $11 \%$ ( $7 \%$ to $21 \%$ ) for not exercising regularly.

When women were given a point for each low risk lifestyle factor they adhered to, increasing the number of low risk factors was significantly and inversely associated with gestational diabetes risk (figure $\downarrow$ ). Compared with women who did not adhere to any low risk lifestyle factors before pregnancy, women who adhered to all four factors had an $83 \%$ lower risk of gestational diabetes (relative risk $0.17,95 \%$ confidence interval 0.12 to 0.25 ).

Tables 3 shows the estimates of the relative risk among women in the combined categories of low risk lifestyle compared with all other women. $\Downarrow$ Women who were in the low risk categories for three lifestyle factors (diet, exercise, and cigarette smoking) had a relative risk of gestational diabetes of 0.59 (95\% confidence interval 0.48 to 0.71 ) compared with all other women. The corresponding population attributable risk percentage was $35 \%$ ( $25 \%$ to $45 \%$ ). The population attributable risk percentage increased to $48 \%$ ( $36 \%$ to $57 \%$ ) when we also included normal included weight status in the definition of low risk lifestyle; however, only $16 \%$ of pregnancies met criteria for the low risk group. A similar population attributable risk percentage (49.2\%) was observed when we applied the distributions of the four low risk factors from the US National Health and Nutrition Examination Survey 2007-10 to the calculation (see appendix 2). 
Results were similar when we restricted the analysis to nulliparous women (relative risk $0.54,95 \%$ confidence interval 0.39 to 0.75 , for all four low risk lifestyle factors) (table $4 \Downarrow$ ). When we examined the association using all pregnancies regardless of previous gestational diabetes status, we observed that the association was slightly attenuated: the relative risk was $0.51(0.41$ to 0.63$)$ with a corresponding population attributable risk percentage of $45.0 \%$ ( $33.9 \%$ to $54.1 \%$ ). We did not observe effect modification by the factors considered, including age, BMI, family history of diabetes, or race/ethnicity (all $\mathrm{P}>0.05$ for heterogeneity). The point estimate for population attributable risk percentage seems much greater among non-white than white women $(79 \% v 45 \%)$. The difference, however, was not significant, and the number of non-white women in this population was small (two non-white women had gestational diabetes events).

\section{Discussion}

In this large prospective cohort study of women of reproductive age, we observed that a low risk lifestyle before pregnancy (that is, maintaining a healthy body weight, consuming a healthy diet, exercising regularly, and not smoking) was strongly and inversely associated with the risk of gestational diabetes. Women at low risk for all four lifestyle factors had more than $80 \%$ lower risk than those without any of the low risk factors. The population attributable risk percentage for smoking, inactivity, overweight or obese, and poor diet in combination was $48 \%$. A similar population attributable risk percentage was observed when we derived it based on distributions of the four low risk factors from the nationally representative US National Health and Nutrition Examination Survey.

\section{Results in relation to other studies and implications of the findings}

There have been few studies on the role of lifestyle factors before pregnancy in the development of gestational diabetes, with most evidence on lifestyle factors or treatment of hyperglycemia during pregnancy after the occurrence of gestational diabetes. Emerging findings from several pilot feasibility studies show evidence for lifestyle interventions in the primary prevention of gestational diabetes with risk factors for gestational diabetes as end points. ${ }^{30}{ }^{31}$ For example, in a pilot and feasibility study of 54 women at high risk for gestational diabetes in Finland, a multi-component lifestyle intervention during pregnancy resulted in a somewhat lower gestational weight gain in the intervention group than the control group, ${ }^{32}$ although there was no impact on glucose tolerance..$^{31}$ In another randomized controlled trial among 50 non-Hispanic white obese pregnant women, the intervention group reduced their total energy intake and had lower fasting glucose and insulin concentrations at 36 weeks' gestation compared with the standard care group..$^{33}$ Evidence from additional studies is necessary to bolster the sparse literature on lifestyle interventions in pregnancy for the prevention of gestational diabetes. Findings from our study, however, suggest that prevention of gestational diabetes could start early, even before pregnancy.

We found that excess body adiposity is the strongest risk factor of gestational diabetes. Obesity has become a global epidemic, and its prevalence continues to increase. Women of reproductive age are no exception. Data from the National Health and Nutrition Examination Survey indicate that $60 \%$ of US women aged 20-39 are overweight or obese..$^{34}$ Moreover, a high proportion of pregnant women, about $45 \%$, are overweight or obese when they become pregnant. ${ }^{35}$ Weight control before pregnancy would be the most effective way to lower the risk of gestational diabetes, but current strategies have not been successful on a population basis. Our data suggest that, even among overweight or obese women, healthy diet and lifestyle was associated with a significantly lower risk of gestational diabetes.

\section{Possible explanations of the findings}

We did not specifically measure lifestyle information during pregnancy. It is plausible that the lower risk of gestational diabetes associated with low risk lifestyle that we observed for the period before pregnancy reflects continued or improved habits during pregnancy. Nonetheless, reduction in risk associated with lifestyle before pregnancy is biologically plausible. During a normal pregnancy, the third trimester is characterized by profound metabolic stresses on maternal lipid and glucose homeostasis, such as marked insulin resistance, hyperinsulinemia, and hyperlipidemia, favoring the transfer of nutrients to the fetus. ${ }^{36}{ }^{37}$ In most pregnant women, however, glucose tolerance stays within the normal range. Women who develop gestational diabetes are thought to have a compromised capacity to adapt to these metabolic challenges of pregnancy. ${ }^{36}$ Pregnancy potentially serves to unmask a predisposition to glucose metabolic disorders in a subset of women. Factors that affect insulin resistance or relative insulin deficiency before pregnancy could thus affect the risk of gestational diabetes. Emerging findings also showed that adipokines and inflammatory markers were higher among women who develop gestational diabetes an average of seven years before the index pregnancy and most affected women have impaired $\beta$ cell function before pregnancy. ${ }^{38}$

\section{Strengths and potential limitations of this study}

Strengths of our study include its prospective cohort design with large numbers of gestational diabetes events and detailed and repeated measurements of diet and lifestyle factors with a previously validated questionnaire. ${ }^{18-20} 23$ To our knowledge, this is the largest and the first prospective cohort study that comprehensively investigated the association of a combination of diet and lifestyle factors before pregnancy with the risk of gestational diabetes.

As in any observational study, measurement error in self reported variables is inevitable; however, misclassification in this prospective study should be non-differential with respect to disease status and would thus have underestimated the true relative risks. We used well validated questionnaires, and the Nurses' Health Study II cohort comprised health professionals who are likely to be more familiar with health related terminology than the general population, minimizing concern for measurement errors and substantial attenuation. ${ }^{192023}$ Additionally, self reported weight was highly correlated with measured weight among a random subset of cohort participants in the Boston area $(\mathrm{r}=0.97) .^{18}$

The overall $5.6 \%$ rate of gestational diabetes in the present study is in the upper range of the usual reported rates based on criteria from the National Diabetes Data group (NDDG). This rate likely reflects the high rate of screening in the cohort. In addition, this estimate of incidence reflects a "per woman reporting 1 or more singleton pregnancy" rather than "per pregnancy" rate. Our validation study indicated a high degree of accuracy of self reported gestational diabetes compared with medical record review. ${ }^{24}$ The number of cases of gestational diabetes in our cohort might be slightly overestimated by over-reporting of 
gestational diabetes. Misclassification of non-cases as cases would be expected only to attenuate observed associations between risk factors and outcome and so would not explain positive results. Conceivably, women with classic risk factors for gestational diabetes such as obesity and advanced age might be screened more often than women without such risk factors, artificially increasing the observed risks associated with such characteristics. Our validation study, however, showed a high level of surveillance for gestational diabetes regardless of risk factor status and noted no significant bias in screening based on risk factor profile. ${ }^{24}$

Interpreting the population attributable risk estimates assumes a causal association between the healthy life style factors and risk of gestational diabetes. This is a big assumption, given that our findings were based on an observational study. An effective long term intervention study on the effects of multiple lifestyle factors before pregnancy for the primary prevention of gestational diabetes, however, would require a large sample size and would be costly and prohibitively challenging to recruit a generalizable study population before conception. Thus far, data from such intervention studies are still lacking. As such, a carefully conducted observational study could provide a reasonable approach to evaluate any association. In addition, we carefully controlled for major well documented risk factors for gestational diabetes to minimize confounding.

The population attributable risk percentage is a population specific calculation contingent on the prevalence of the exposure and its association with risk of disease. The percentage estimated from our cohort of parous health professionals probably underestimates the burden of unhealthy behaviors on the risk of gestational diabetes in the general population because the prevalence of these unhealthy behaviors in the Nurses' Health Study II is probably lower. For instance, the prevalence of overweight or obese among US women of reproductive age was about $60 \%$ in the National Health and Nutrition Examination Survey $2007-08^{33}$ but only $27 \%$ in the present study. When we derived the population attributable risk percentage based on the distributions of the four low risk factors according to the recent prevalence among women of reproductive ages (ages 18-45) in the nationally representative US National Health and Nutrition Examination Survey (2007-10), we found a similar but slightly higher percentage. Nonetheless, future studies among non-white populations are warranted.

\section{Conclusions}

In conclusion, our data indicate that adherence to a healthful lifestyle in the period before pregnancy is associated with a substantially lower risk of gestational diabetes. Maintaining a healthy body weight throughout one's reproductive life would confer the greatest benefit. A healthy diet and regular exercise were associated with a substantial lower risk, independent of their benefit on body weight. Importantly, among both normal weight and overweight/obese women a healthy lifestyle was related to a lower risk. Our findings also highlight the potential benefit of integrating lifestyle counseling into preconception care. Although it is always challenging to change behavior/lifestyle, the time before and during pregnancy could represent an opportunity to change diet and lifestyle as these women might be particularly motivated to adhere to advice to improve pregnancy and/or birth outcomes.

Contributors: $\mathrm{CZ}$ designed the study. $\mathrm{FH}$ was involved in data collection. DKT and DW provided statistical expertise. DKT and DW analyzed the data. $\mathrm{CZ}$ wrote the first draft of the manuscript. All authors contributed to the interpretation of the results and revision of the manuscript for important intellectual content and approved the final version of the manuscript. $\mathrm{CZ}$ and $\mathrm{FH}$ are guarantors.

Funding: This study was supported by the intramural research program of the Eunice Kennedy Shriver National Institute of Child Health and Human Development, National Institutes of Health (contract No HHSN275201000020C). The Nurses' Health Study II was funded by research grants DK58845, CA50385, P30 DK46200, and UM1 CA176726 from the National Institutes of Health and by an American Diabetes Association Award 7-12-MN-34.

Competing interest: All authors have completed the ICMJE uniform disclosure form at www.icmje.org/coi_disclosure.pdf and declare: no support from any organization for the submitted work; no financial relationships with any organizations that might have an interest in the submitted work in the previous three years; no other relationships or activities that could appear to have influenced the submitted work Ethical approval: The study protocol was approved by the institutional review boards of the Partners Health Care System (Boston, MA, USA). The completion of the self administered questionnaire was considered to imply informed consent.

Transparency declaration: The lead author, $\mathrm{CZ}$, affirms that the manuscript is an honest, accurate, and transparent account of the study being reported; that no important aspects of the study have been omitted. Data sharing: No additional data available.

1 American Diabetes Association. Gestational diabetes mellitus. Diabetes Care 2004;27(suppl 1):S88-90.

2 Hartling L, Dryden DM, Guthrie A, Muise M, Vandermeer B, Aktary WM, et al. Screening and diagnosing gestational diabetes mellitus. (Evidence Reports/Technology Assessments No 210.) Agency for Healthcare Research and Quality, 2012.

3 Ferrara A, Kahn HS, Quesenberry CP, Riley C, Hedderson MM. An increase in the incidence of gestational diabetes mellitus: Northern California, 1991-2000. Obstet Gynecol 2004;103:526-33.

4 Dabelea D, Snell-Bergeon JK, Hartsfield CL, Bischoff KJ, Hamman RF, McDuffie RS. Increasing prevalence of gestational diabetes mellitus (GDM) over time and by birth cohort: Kaiser Permanente of Colorado GDM Screening Program. Diabetes Care 2005;28:579-84.

5 Zhang F, Dong L, Zhang CP, Li B, Wen J, Gao W, et al. Increasing prevalence of gestational diabetes mellitus in Chinese women from 1999 to 2008. Diabet Med 2011;28:652-7.

6 Reece EA, Leguizamon G, Wiznitzer A. Gestational diabetes: the need for a common ground. Lancet 2009;373:1789-97.

7 Bryson CL, loannou GN, Rulyak SJ, Critchlow C. Association between gestational diabetes and pregnancy-induced hypertension. Am J Epidemiol 2003;158:1148-53.

8 Bellamy L, Casas JP, Hingorani AD, Williams D. Type 2 diabetes mellitus after gestational diabetes: a systematic review and meta-analysis. Lancet 2009;373:1773-9.

9 Mitanchez D. Foetal and neonatal complications in gestational diabetes: perinatal mortality, congenital malformations, macrosomia, shoulder dystocia, birth injuries, neonatal complications. Diabetes Metabol 2010;36:617-27.

10 Hillier TA, Pedula KL, Schmidt MM, Mullen JA, Charles MA, Pettitt DJ. Childhood obesity and metabolic imprinting: the ongoing effects of maternal hyperglycemia. Diabetes Care 2007;30:2287-92.

11 Clausen TD, Mathiesen ER, Hansen T, Pedersen O, Jensen DM, Lauenborg J, et al. High prevalence of type 2 diabetes and pre-diabetes in adult offspring of women with gestational diabetes mellitus or type 1 diabetes: the role of intrauterine hyperglycemia. Diabetes Care 2008;31:340-6.

12 Egeland GM, Skjaerven R, Irgens LM. Birth characteristics of women who develop gestational diabetes: population based study. BMJ 2000;321:546-7.

13 Zhang $C$, Ning Y. Effect of dietary and lifestyle factors on the risk of gestational diabetes: review of epidemiologic evidence. Am J Clin Nutr 2011:94:1975S-79S.

14 Solomon CG, Willett WC, Carey VJ, Rich-Edwards J, Hunter DJ, Colditz GA, et al. A prospective study of pregravid determinants of gestational diabetes mellitus. JAMA 1997;278:1078-83.

15 Tobias DK, Zhang C, Chavarro J, Bowers K, Rich-Edwards J, Rosner B, et al. Prepregnancy adherence to dietary patterns and lower risk of gestational diabetes mellitus. Am J Clin Nutr 2012:96:289-95.

16 Zhang C, Solomon CG, Manson JE, Hu FB. A prospective study of pregravid physical activity and sedentary behaviors in relation to the risk for gestational diabetes mellitus. Arch Intern Med 2006;166:543-8.

17 Bao W, Tobias DK, Bowers K, Chavarro J, Vaag A, Grunnet LG, et al. Physical activity and sedentary behaviors in relation to the risk of progression from gestational diabetes to type 2 diabetes: a prospective cohort study. JAMA Intern Med 2014:174:1047-55.

18 Rimm EB, Stampfer MJ, Colditz GA, Chute CG, Litin LB, Willett WC. Validity of self-reported waist and hip circumferences in men and women. Epidemiology 1990;1:466-73.

19 Salvini S, Hunter DJ, Sampson L, Stampfer MJ, Colditz GA, Rosner B, et al. Food-based validation of a dietary questionnaire: the effects of week-to-week variation in food consumption. Int J Epidemiol 1989:18:858-67.

20 Willett WC, Sampson L, Browne ML, Stampfer MJ, Rosner B, Hennekens CH, et al. The use of a self-administered questionnaire to assess diet four years in the past. $A m \mathrm{~J}$ Epidemiol 1988;127:188-99.

21 Chiuve SE, Fung TT, Rimm EB, Hu FB, McCullough ML, Wang M, et al. Alternative dietary indices both strongly predict risk of chronic disease. J Nutr 2012;142:1009-18.

22 Kennedy ET, Ohls J, Carlson S, Fleming K. The Healthy Eating Index: design and applications. J Am Diet Assoc 1995;95:1103-8. 


\section{What is already known on this topic}

Gestational diabetes is a common complication of pregnancy and is at the center of a vicious trans-generational circle of diabetes-begets-diabetes

Several potentially modifiable risk factors before pregnancy have been identified for gestational diabetes

\section{What this study adds}

Adherence to a low risk lifestyle before pregnancy is associated with a substantial lower risk of gestational diabetes Such findings apply to both normal weight and overweight/obese women

23 Wolf AM, Hunter DJ, Colditz GA, Manson JE, Stampfer MJ, Corsano KA, et al. Reproducibility and validity of a self-administered physical activity questionnaire. Int $J$ Epidemiol 1994;23:991-9.

24 Solomon CG, Willett WC, Rich-Edwards J, Hunter DJ, Stampfer MJ, Colditz GA, et al. variability in diagnostic evaluation and criteria for gestational diabetes. Diabetes Care 1996;19:12-6.

25 WHO Expert Committee. Physical status: the use and interpretation of anthropometry. World Health Organ Tech Rep Ser 1995;854:1-452.

26 Physical Activity Guidelines Committee. Physical activity guidelines advisory committee report. Department of Health and Human Services, 2008.

27 Hanley JA, Negassa A, Edwardes MD, Forrester JE. Statistical analysis of correlated data using generalized estimating equations: an orientation. Am J Epidemiol 2003;157:364-75

28 Spiegelman D, Hertzmark E, Wand HC. Point and interval estimates of partial population attributable risks in cohort studies: examples and software. Cancer Cause Control 2007:18:571-9.

29 Wacholder S, Benichou J, Heineman EF, Hartge P, Hoover RN. Attributable risk: advantages of a broad definition of exposure. Am J Epidemiol 1994;140:303-9.

30 Luoto R, Kinnunen TI, Aittasalo M, Kolu P, Raitanen J, Ojala K, et al. Primary prevention of gestational diabetes mellitus and large-for-gestational-age newborns by lifestyle counseling: a cluster-randomized controlled trial. PLoS Med 2011;8:e1001036.

31 Korpi-Hyovalti E, Schwab U, Laaksonen DE, Linjama H, Heinonen S, Niskanen L. Effect of intensive counselling on the quality of dietary fats in pregnant women at high risk of gestational diabetes mellitus. Br J Nutr 2012;108:910-7.

32 Korpi-Hyovalti E, Heinonen S, Schwab U, Laaksonen DE, Niskanen L. Effect of intensive counselling on physical activity in pregnant women at high risk for gestational diabetes mellitus. A clinical study in primary care. Primary Care Diabetes 2012;6:261-8.
33 Wolff S, Legarth J, Vangsgaard K, Toubro S, Astrup A. A randomized trial of the effects of dietary counseling on gestational weight gain and glucose metabolism in obese pregnant women. Int J Obes (Lond) 2008;32:495-501.

34 Flegal KM, Carroll MD, Ogden CL, Curtin LR. Prevalence and trends in obesity among US adults, 1999-2008. JAMA 2010;303:235-41.

35 Gunderson EP. Childbearing and obesity in women: weight before, during, and after pregnancy. Obstet Gynecol Clin North Am 2009;36:317-32.

36 Buchanan TA, Xiang AH. Gestational diabetes mellitus. J Clin Invest 2005;115:485-91.

37 Kaaja RJ, Greer IA. Manifestations of chronic disease during pregnancy. JAMA 2005;294:2751-7.

38 Hedderson MM, Darbinian J, Havel PJ, Quesenberry CP, Sridhar S, Ehrlich S, et al. Low Prepregnancy adiponectin concentrations are associated with a marked increase in risk for development of gestational diabetes mellitus. Diabetes Care 2013;36:3930-7.

Accepted: 18 August 2014

\section{Cite this as: BMJ 2014;349:95450}

This is an Open Access article distributed in accordance with the Creative Commons Attribution Non Commercial (CC BY-NC 3.0) license, which permits others to distribute, remix, adapt, build upon this work non-commercially, and license their derivative works on different terms, provided the original work is properly cited and the use is non-commercial. See: http://creativecommons.org/licenses/by-nc/3.0/. 


\section{Tables}

Table 1| Relative risk of gestational diabetes by individual modifiable risk factors before pregnancy in 20136 pregnancies in Nurses' Health Study II

\begin{tabular}{|c|c|c|}
\hline & No $(\%)$ of pregnancies & Relative risk $(95 \% \mathrm{Cl})^{*}$ \\
\hline \multicolumn{3}{|c|}{ Alternate Healthy Eating Index-2010 diet score (fifth): } \\
\hline 1 & $4017(20.0)$ & 1.00 (reference) \\
\hline 2 & $4106(20.4)$ & $0.96(0.79$ to 1.17$)$ \\
\hline 3 & 3917 (19.5) & $0.98(0.80$ to 1.21$)$ \\
\hline 4 & $3877(19.3)$ & $0.84(0.67$ to 1.04$)$ \\
\hline 5 & $4219(21.0)$ & 0.75 (0.59 to 0.94$)$ \\
\hline \multicolumn{3}{|c|}{ Moderate/vigorous activity (min/week): } \\
\hline$<30$ & $5080(25.2)$ & 1.00 (reference) \\
\hline $30-59$ & $2239(11.1)$ & $0.90(0.72$ to 1.13$)$ \\
\hline 60-89 & $1937(9.6)$ & $0.91(0.71$ to 1.16$)$ \\
\hline $90-149$ & $2596(12.9)$ & $0.89(0.71$ to 1.10$)$ \\
\hline $150-209$ & $2111(10.5)$ & $0.85(0.66$ to 1.10$)$ \\
\hline$\geq 210$ & $6173(30.7)$ & $0.78(0.64$ to 0.94$)$ \\
\hline \multicolumn{3}{|l|}{ BMI: } \\
\hline$<18.5$ & $743(3.7)$ & 1.18 (0.72 to 1.94$)$ \\
\hline 18.5-19.9 & $2438(12.1)$ & 1.00 (reference) \\
\hline $20.0-22.9$ & $7851(39.0)$ & $1.10(0.82$ to 1.48$)$ \\
\hline $23.0-24.9$ & $3600(17.9)$ & 1.61 (1.19 to 2.19$)$ \\
\hline $25.0-26.9$ & 2061 (10.2) & 2.14 (1.55 to 2.93$)$ \\
\hline 27.0-28.9 & $1183(5.9)$ & $2.20(1.56$ to 3.12$)$ \\
\hline $29.0-30.9$ & $752(3.7)$ & 2.58 (1.78 to 3.75$)$ \\
\hline $31.0-32.9$ & $571(2.8)$ & $3.86(2.71$ to 5.50$)$ \\
\hline $33.0-34.9$ & $327(1.6)$ & 4.38 (2.98 to 6.43$)$ \\
\hline$\geq 35.0$ & $610(3.0)$ & 4.45 (3.19 to 6.20$)$ \\
\hline \multicolumn{3}{|l|}{ Smoking status: } \\
\hline Never smoker & $14543(72.2)$ & 1.00 (reference) \\
\hline Former smoker & 3977 (19.8) & 1.09 (0.91 to 1.29$)$ \\
\hline Current smoker & $1616(8.0)$ & $1.43(1.17$ to 1.76$)$ \\
\hline
\end{tabular}

BMI=body mass index.

*Adjusted for age, other variables included in table, parity, family history of diabetes, history of infertility, race/ethnicity, questionnaire period, total energy intake, and alcohol intake. 
Table 2| Low risk lifestyle factors and risk of gestational diabetes in 20136 pregnancies in Nurses' Health Study II

\begin{tabular}{|c|c|c|c|c|}
\hline & \multicolumn{2}{|c|}{ No $(\%)$ of pregnancies } & \multirow[b]{2}{*}{ Relative risk* $(95 \% \mathrm{Cl})$} & \multirow{2}{*}{$\begin{array}{c}\text { Population attributable risk } \\
\text { percentage }+(95 \% \mathrm{Cl})\end{array}$} \\
\hline & Low risk group & Gestational diabetes & & \\
\hline Current non-smoker & $18520(92.0)$ & 727 & 0.71 (0.58 to 0.87 ) & 3.4 (1.5 to 4.9$)$ \\
\hline $\mathrm{BMI}<25$ & $14632(72.7)$ & 410 & 0.44 (0.38 to 0.50$)$ & 28.1 (25.1 to 31.1 ) \\
\hline Healthy dietł & $8096(40.2)$ & 284 & 0.81 (0.70 to 0.94$)$ & 12.4 (3.9 to 19.6$)$ \\
\hline $\begin{array}{l}\text { Moderate/vigorous exercise } \\
\geq 150 \mathrm{~min} / \text { week }\end{array}$ & $8284(41.1)$ & 270 & 0.85 (0.73 to 0.99$)$ & $10.1(0.7$ to 18.1$)$ \\
\hline
\end{tabular}

*Adjusted for age, other variables included in table, parity, family history of diabetes, history of infertility, race/ethnicity, questionnaire period, total energy intake, and alcohol intake. Reference group for relative risk calculation is all other pregnancies not in low risk factor category as defined in table-for instance, relative risk comparing $\mathrm{BMI}<25$ with $\mathrm{BMI} \geq 25$ is 0.44 .

†Percentage of gestational diabetes cases in population theoretically attributable to non-adherence to particular factor.

$\ddagger$ Alternate Healthy Eating Index-2010 diet score in upper two fifths. 
Table 3| Combined low risk lifestyle factors and risk of gestational diabetes in 20136 pregnancies in Nurses' Health Study II

\begin{tabular}{|c|c|c|c|c|}
\hline Low risk group & $\begin{array}{l}\text { Percentage of } \\
\text { pregnancies }\end{array}$ & $\begin{array}{l}\text { No of pregnancies with } \\
\text { gestational diabetes }\end{array}$ & Relative risk* $(95 \% \mathrm{Cl})$ & $\begin{array}{l}\text { Population attributable risk } \\
\text { percentage }(95 \% \mathrm{Cl})\end{array}$ \\
\hline $\begin{array}{l}3 \text { factors in low risk category (current } \\
\text { non-smoker, moderate/vigorous physical } \\
\text { activity } \geq 150 \mathrm{~min} / \text { week, healthy eating } \ddagger \text { ) }\end{array}$ & 20.3 & 112 & $0.59(0.48$ to 0.71$)$ & 35.4 (25.1 to 44.9$)$ \\
\hline $\begin{array}{l}\text { All } 4 \text { factors in low risk category (BMI } \\
<25.0 \text {, current non-smoker, } \\
\text { moderate/vigorous physical activity } \geq 150 \\
\text { min/week, healthy eating } \ddagger \text { ) }\end{array}$ & 16.3 & 71 & $0.48(0.38$ to 0.61$)$ & 47.5 (35.6 to 56.6 ) \\
\hline
\end{tabular}

*Estimated from generalized estimating equation models and adjusted for age, parity, family history of diabetes, history of infertility, race/ethnicity, questionnaire period, total energy intake, and alcohol intake. Reference group for relative risk is all other pregnancies not in low risk group as defined in table. †Percentage of cases of gestational diabetes in population theoretically attributable to non-adherence to particular factors. $\ddagger$ Alternate Healthy Eating Index-2010 diet score in upper two fifths. 
Table 4| Low risk healthy lifestyle* and risk of gestational diabetes stratified by diabetes risk factors in 20136 pregnancies in Nurses' Health Study II

\begin{tabular}{|c|c|c|c|c|}
\hline & $\begin{array}{l}\text { Percentage of pregnancies } \\
\text { in each group }\end{array}$ & $\begin{array}{l}\text { No of pregnancies with } \\
\text { gestational diabetes }\end{array}$ & $\begin{array}{l}\text { Relative risk } \dagger \\
(95 \% \mathrm{Cl})\end{array}$ & $\begin{array}{l}\text { Population attributable risk } \\
\text { percentage } \neq(95 \% \mathrm{Cl})\end{array}$ \\
\hline \multicolumn{5}{|c|}{ Family history of diabetes: } \\
\hline Yes & 13.3 & 16 & $0.52(0.31$ to 0.86$)$ & 44.2 (12.9 to 63.6$)$ \\
\hline No & 16.7 & 55 & 0.47 (0.36 to 0.62$)$ & 48.3 (34.6 to 58.3 ) \\
\hline \multicolumn{5}{|l|}{ Parity: } \\
\hline Nulliparous & 22.5 & 39 & $0.54(0.39$ to 0.75$)$ & $40.3(21.9$ to 53.4$)$ \\
\hline Parous & 13.9 & 32 & 0.44 (0.31 to 0.63$)$ & 52.5 (34.7 to 64.7 ) \\
\hline \multicolumn{5}{|l|}{ Age (years): } \\
\hline$<35$ & 15.7 & 29 & 0.49 (0.33 to 0.71$)$ & 46.5 (26.5 to 61.1$)$ \\
\hline$\geq 35$ & 16.9 & 42 & $0.47(0.35$ to 0.64$)$ & 48.5 (32.9 to 59.5$)$ \\
\hline \multicolumn{5}{|l|}{ Race: } \\
\hline White & 16.3 & 69 & 0.51 (0.40 to 0.65$)$ & 44.5 (31.8 to 54.5$)$ \\
\hline Non-white & 15.8 & 2 & $0.19(0.05$ to 0.74$)$ & 78.7 (25.2 to 92.2 ) \\
\hline \multicolumn{5}{|c|}{ Overweight (BMI $\geq 25)$ : } \\
\hline Yes & 14.7 & 41 & 0.60 (0.43 to 0.82$)$ & $36.0(16.2$ to 51.3$)$ \\
\hline No & 22.4 & 71 & 0.66 (0.51 to 0.86$)$ & 28.1 (11.6 to 40.5$)$ \\
\hline
\end{tabular}

*Having all four factors in low risk category (current non-smoker, BMI <25.0, moderate/vigorous physical activity >150 min/week, Alternate Healthy Eating Index-2010 diet score in upper two fifths), except for stratified analysis by overweight status in which other three factors except BMI were included.

†Adjusted for age, parity, family history of diabetes, history of infertility, race/ethnicity, questionnaire period, total energy intake, and alcohol intake. Reference group is all other pregnancies not having all four low risk factors except for stratified analysis by overweight status in which reference group is all other pregnancies not having other three low risk factors.

$\ddagger$ Percentage of gestational diabetes cases in population theoretically attributable to non-adherence to particular factor. 


\section{Figure}

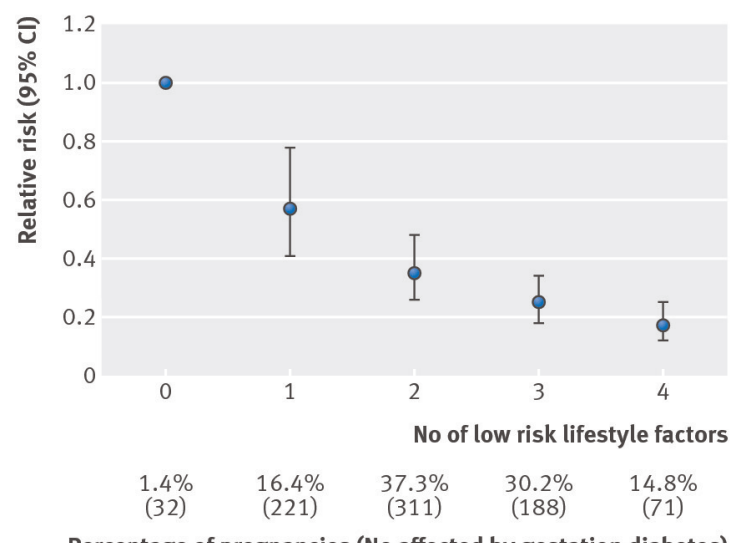

Percentage of pregnancies (No affected by gestation diabetes)

Association between number of low risk lifestyle factors and risk of gestational diabetes. Low risk lifestyle factors include maintaining healthy body weight, consuming healthy diet, regular exercise, and not smoking. Relative risk adjusted for age, parity, family history of diabetes, history of infertility, race/ethnicity, questionnaire period, total energy intake, and alcohol consumption 